\title{
NONLINEAR STABILITY PROBLEM OF A ROTATING POROUS LAYER
}

\author{
BY \\ Y. QIN AND P. N. KALONI \\ Department of Mathematics and Statistics, University of Windsor, Windsor, Ontario, Canada
}

1. Introduction. In hydrodynamic stability theory there are two useful methods, namely, the linear stability method and the energy method. Both of these methods have been used extensively, and, in fact, complement each other. While the linear stability method determines a critical dimensionless bound above which the disturbances of a basic flow are unstable, the energy method predicts a critical bound below which a basic flow is asymptotically stable (in the Liapunov sense). However, in some cases when the linearized system of governing equations happens to be selfadjoint, the linear stability and energy stability predictions coincide (cf. Davis, 1969, Galdi and Straughan, 1985a).

The energy method is essentially due to Orr (1907), but its recent revival has been inspired by the creative work of Serrin (1959) and Joseph $(1965,1966)$. Despite the unquestionable success of the energy method in several stability problems, there is some skepticism about its ongoing indiscriminate use. Situations have been encountered, for example, in the magnetic Bénard problem (Rionero 1968, Galdi 1985 ) and in the Bénard problem between rotating parallel, horizontal planes (Galdi and Straughan 1985b) where the classical energy theory of Serrin and Joseph did not produce expected results. Rapid improvements of the classical energy theory, however, have been made by Galdi and Straughan (1985b), Mulone and Rionero (1989), and others by proposing generalized energy functionals. This new approach to energy theory is summarized and further analysed by Galdi and Padula (1990). In this remarkable paper, the authors have singled out systems for which the new theory furnishes necessary and sufficient conditions for stability, and have provided an algorithm to construct the appropriate generalized energy functionals for a wide class of physical problems. With regard to the rotating Bénard problem, we note that an energy function using only the $L^{2}$ norm of the disturbance variables may not be good enough to obtain the desired results. The generalized energy functionals, with stronger norm, usually $W^{1,2}$ or an equivalent one, would be useful in the study of the nonlinear stability of the rotating Benard problem.

The stability problems of convective flow in porous media using the energy method have been investigated by many authors: Westbrook (1969) applied the energy method to discuss the stability of convective flow in a porous medium, and Homsy and 
Sherwood (1976) extended this work when there is a mass flow. Joseph (1976), using the energy method, considered the problem of finding the critical Rayleigh number for a fluid-saturated porous layer which is subjected to an internal heat source. His results have been extended by Rionero and Straughan (1990) by allowing variable gravity effects in their calculations. In all the above papers the porous medium is represented by Darcy's model. When the porosity of the material is large and when the boundary layer effects need to be taken into consideration, it is now believed that the Brinkman model is superior to Darcy's model. Moreover, use of this model allows us to recover, in the limiting cases, results for flow of pure viscous fluid as well as for flow governed by Darcy's law. Qin and Kaloni (1992) have carried out a theoretical study of the problem of steady convection through porous media based upon the Brinkman model.

In this work we study the nonlinear stability of the rotating Bénard problem in a porous medium. We employ the generalized Brinkman model as a suitable prototype for high porosity porous media, and, based on it, we are able to apply the energy theory of Galdi and Padula (1990) in studying this problem.

In this paper, after presenting the basic pertubation equations, the evolution equation of an energy functional is derived. Adopting the same energy functional as constructed by Galdi and Padula for the viscous rotating Bénard problem, the a priori estimate of the energy functional is then given which ensures the nonlinear stability under some sufficient conditions. We then solve the variational problem and carry out numerical calculations to determine the critical energy bounds.

2. Perturbation equations. Let us consider an infinite horizontal porous layer saturated with a homogeneous fluid under the action of a vertical gravity field $\mathbf{g}=-g \mathbf{k}$ in which an adverse temperature gradient $\beta>0$ is maintained; $\mathbf{k}=(0,0,1)$ is the vertical unit vector. We also assume that the fluid is rotating about the vertical axis $z$ with a constant angular velocity $\Omega$. A perturbation of velocity $\mathbf{u}=(u, v, w)$, temperature $\theta$, and pressure field $p$ is taken about the nonconvecting stationary solution $\mathbf{u}=0, T=-\beta z+T_{0}$. The fluid is assumed to be contained in a porous medium between two planes $z=0$ and $z=h$ with assigned temperatures $T=T_{0}$ at $z=0$ and $T=T_{1}$ at $z=h$, where $T_{0}>T_{1}$ and the temperature gradient across the fluid layer is $\beta=\left(T_{0}-T_{1}\right) / h$.

The momentum, continuity, and energy equations for the porous fluid layer in the Boussinesq approximation are, respectively (Walker and Homsy, 1977)

$$
\begin{gathered}
\mathbf{u}_{t}+\frac{1}{\phi} \mathbf{u} \cdot \nabla \mathbf{u}=\Delta \mathbf{u}-P_{0} \mathbf{u}+R \theta \mathbf{k}+T \mathbf{u} \times \mathbf{k}-\nabla p, \\
\nabla \cdot \mathbf{u}=0, \\
P_{r}\left(\theta_{t}+\mathbf{u} \cdot \nabla \theta\right)=\Delta \theta+R w,
\end{gathered}
$$

where $(\mathbf{x}, t, \mathbf{u}, p, T)$ have been made dimensionless with respect to $\left(h, h^{2} / \nu\right.$, $\left.\nu / h, \rho_{0} \nu^{2} / h^{2},\left(\frac{\beta \nu^{3}}{\alpha g \kappa h^{2}}\right)^{1 / 2}\right)$ respectively, $h$ is the fluid depth, $\nu$ is the kinematic viscosity, $\rho_{0}$ is the density, $\kappa$ is the thermal diffusivity, $\alpha$ is the coefficient of 
thermal expansion, $\phi$ is the porosity, $\beta=\left(T_{0}-T_{1}\right) / h$ is the temperature gradient, and the following dimensionless numbers are defined:

$$
\begin{aligned}
R^{2} & =\alpha \beta g h^{2} /(\kappa \nu) & & \text { (Rayleigh number), } \\
T & =2 h^{2} \Omega / \nu & & \text { (Taylor number), } \\
P_{r} & =\nu / \kappa & & \text { (Prandtl number), } \\
P_{0} & =h^{2} / k & & \text { (Darcy number). }
\end{aligned}
$$

Equation (1) is called the Darcy-Brinkman-Boussinesq equation involving convective inertia, the usual viscous force, as well as Darcy resistance force, and Boussinesq approximation in the body force.

For the boundary conditions for the functions $\mathbf{u}, \theta$, we suppose that $\mathbf{u}, \theta, p$ are periodic in $x, y$ with period $2 a_{1}, 2 a_{2}$, respectively, and are stress-free at the surfaces $z=0,1$, so that

$$
u,_{z}=v,_{z}=w=\theta=0, \quad z=0,1,
$$

where $u,_{z}=\partial u / \partial z$.

To exclude rigid motions we assume that the mean values of $u, v$ are zero, i.e., we require

$$
\int_{\Omega} u d x=\int_{\Omega} v d x=0
$$

where $\Omega=\left(0,2 a_{1}\right] \times\left(0,2 a_{2}\right] \times(0,1)$.

3. An energy functional and related evolution equation. Let $u$ be any of the four components of the vector of the perturbation $(\mathbf{u}, \theta)$. The Hilbert space $H$ coincides with $\mathbf{J}(\Omega) \times L^{2}(\Omega)$, and $\mathbf{J}(\Omega)$ is the subspace of $\mathbf{L}^{2}(\Omega)$ consisting of solenoidal vector functions $\mathbf{u}$ with $w=0$ at $z=0,1$.

The problem described by (1) - (5) can be written as an evolution equation of the form

$$
B u_{t}=A u+R S u+T M u+N u
$$

in the Hilbert space $H$, in which $B$ is a positive diagonal matrix, $A$ is a linear differential operator, $S$ is a bounded symmetic operator, $M$ is a skew-symmetric operator, $N$ is a nonlinear operator, and $R$ and $T$ are two dimensionless parameters. Following Galdi and Padula (1990), it can be shown that this system is weakly coupled. Besides the usual energy term, an appropriate energy functional is then proposed by considering the interaction between $S$ and $M$. Rather than verifying all the hypotheses for weakly coupled systems, here we prefer to derive the evolution equation more directly. The procedure followed below, however, is patterned after the work of Galdi and Padula (1990).

Let $\Pi$ be a projector operator from $\mathbf{L}^{2}(\Omega)$ onto $\mathbf{J}(\Omega)$; the following properties hold:

$$
\begin{array}{ll}
\text { 1) } \Pi \mathbf{v}=\mathbf{v}, & \text { if } \mathbf{v} \in \mathbf{J}(\Omega), \\
\text { 2) } \Pi \mathbf{v}=\mathbf{v}-\nabla \psi, & \text { if } \mathbf{v} \in \mathbf{L}^{2}(\Omega) \backslash \mathbf{J}(\Omega),
\end{array}
$$


with $\psi$ (periodic in $x, y$, depending on $\mathbf{v}$ ) satisfying the problem

$$
\begin{aligned}
& \Delta \psi=\nabla \cdot \mathbf{v}, \\
& \psi_{, z}=\mathbf{v} \cdot \mathbf{k} \text { at } z=1,0 .
\end{aligned}
$$

For $(\mathbf{u}, \theta) \in\left(\mathbf{C}^{2}(\overline{\mathbf{\Omega}}) \times C^{2}(\bar{\Omega})\right) \cap\left(\mathbf{J}(\Omega) \times L^{2}(\Omega)\right)$, multiplying (1) by $\mathbf{u}$, (3) by $\theta$, integrating over $\Omega$, and using (4), we have

$$
\begin{aligned}
\frac{1}{2} \frac{d}{d t}\|\mathbf{u}\|^{2} & =-\|\nabla \mathbf{u}\|^{2}-P_{0}\|u\|^{2}+R\langle\theta w\rangle, \\
\frac{1}{2} P_{r} \frac{d}{d t}\|\theta\|^{2} & =-\|\nabla \theta\|^{2}+R\langle\theta w\rangle,
\end{aligned}
$$

where $\|\cdot\|$ denotes the $\mathbf{L}^{2}(\Omega)$ (or $L^{2}(\Omega)$ ) norm, and $\langle\cdot\rangle$ denotes the integral over $\Omega$.

Multiplying (1) by $\Pi(\mathbf{k} \times \Pi(\mathbf{u} \times \mathbf{k}))$, and then by $\Pi(\mathbf{k} \times \Pi(\theta \mathbf{k}))$ respectively, integrating over $\Omega$, we obtain

$$
\begin{aligned}
\frac{1}{2} \frac{d}{d t}\|\Pi(\mathbf{u} \times \mathbf{k})\|^{2}= & -\left\|\mathbf{u},{ }_{,}\right\|^{2}-P_{0}\|\Pi(\mathbf{u} \times \mathbf{k})\|^{2}+R\langle\theta \mathbf{k} \cdot \Pi(\mathbf{k} \times \Pi(\mathbf{u} \times \mathbf{k}))\rangle \\
& -\frac{1}{\phi}\langle(\mathbf{u} \cdot \nabla \mathbf{u}) \cdot \Pi(\mathbf{k} \times \Pi(\mathbf{u} \times \mathbf{k}))\rangle, \\
\left\langle\Pi(\mathbf{u} \times \mathbf{k})_{t} \cdot(\theta \mathbf{k})\right\rangle= & -\left\langle\operatorname{curl}(\mathbf{u} \times \mathbf{k}) \cdot \operatorname{curl}(\theta \mathbf{k})>-P_{0}<\Pi(\mathbf{u} \times \mathbf{k}) \cdot(\theta \mathbf{k})\right\rangle \\
+ & T\langle(\mathbf{u} \times \mathbf{k}) \cdot \Pi(\mathbf{k} \times \Pi(\theta \mathbf{k}))\rangle-\frac{1}{\phi}\langle(\mathbf{u} \cdot \nabla \mathbf{u}) \cdot \Pi(\mathbf{k} \times \Pi(\theta \mathbf{k}))\rangle,
\end{aligned}
$$

where the properties in (6) and (7) are used to derive these equations.

Multiplying (3) by $\Pi(\mathbf{u} \times \mathbf{k}) \cdot \mathbf{k}$ and integrating over $\Omega$, we also have

$$
\begin{aligned}
P_{r}\left\langle\theta_{t} \Pi(\mathbf{u} \times \mathbf{k}) \cdot \mathbf{k}\right\rangle= & -\langle\operatorname{curl}(\mathbf{u} \times \mathbf{k}) \cdot \operatorname{curl}(\theta \mathbf{k})\rangle+R\langle\Pi(\mathbf{u} \times \mathbf{k}) \cdot \mathbf{k} w\rangle \\
& -P_{r}\langle(\mathbf{u} \cdot \nabla \theta) \Pi(\mathbf{u} \times \mathbf{k}) \cdot \mathbf{k}\rangle .
\end{aligned}
$$

Finally, multiplying (1) by $\Delta \mathbf{u},(3)$ by $\Delta \theta$, and integrating over $\Omega$, we get

$$
\begin{gathered}
\frac{1}{2} \frac{d}{d t}\|\nabla \mathbf{u}\|^{2}=-\|\Delta \mathbf{u}\|^{2}-P_{0}\|\nabla \mathbf{u}\|^{2}-R\langle\Delta w \theta\rangle+\frac{1}{\phi}\langle(\mathbf{u} \cdot \nabla) \mathbf{u} \cdot \Delta \mathbf{u}\rangle, \\
\frac{1}{2} P_{r} \frac{d}{d t}\|\nabla \theta\|^{2}=-\|\Delta \theta\|^{2}-R\langle\Delta \theta w\rangle-P_{r}\langle\mathbf{u} \cdot \nabla \theta \Delta \theta\rangle .
\end{gathered}
$$

We now choose the same energy functional as constructed by Galdi and Padula (1990, p. 246)

$$
\begin{gathered}
E=\frac{1}{2}\left(\|\mathbf{u}\|^{2}+P_{r}\|\theta\|^{2}+\lambda_{2}\|\Pi(\mathbf{u} \times \mathbf{k})\|^{2}+2 \lambda P_{r}^{\frac{1}{2}}\langle\Pi(\mathbf{u} \times \mathbf{k}) \cdot \theta \mathbf{k}\rangle\right), \\
E_{1}=\frac{1}{2}\left(\|\mathbf{u}\|^{2}+P_{r}\|\theta\|^{2}+\|\nabla \mathbf{u}\|^{2}+P_{r}\|\nabla \theta\|^{2}\right),
\end{gathered}
$$

and write

$$
V=E+\eta E_{1}
$$

where $\lambda, \lambda_{2}, \eta$ are the positive coupling parameters. To ensure that $E$ is positivedefinite, the condition

$$
\lambda_{2}>\lambda^{2}
$$


must be imposed. Using (15)-(17), together with all the results in (8)-(14), we arrive at

$$
d V / d t=F(u)-D(u)+N(u)+\eta\left(F_{1}(u)-D_{1}(u)+N_{1}(u)\right),
$$

where

$$
\begin{aligned}
F(u)= & 2 R\langle\theta w\rangle-\lambda P_{r}^{1 / 2}\left(1+1 / P_{r}\right)\langle\operatorname{curl}(\mathbf{u} \times \mathbf{k}) \cdot \operatorname{curl}(\theta \mathbf{k})\rangle \\
& -\lambda P_{0} P_{r}^{1 / 2}\langle\Pi(\mathbf{u} \times \mathbf{k}) \cdot \theta \mathbf{k}\rangle+\lambda \frac{R}{P_{r}^{1 / 2}}\langle\Pi(\mathbf{u} \times \mathbf{k}) \cdot \mathbf{k} w\rangle \\
& +\lambda P_{r}^{1 / 2} T\langle(\mathbf{u} \times \mathbf{k}) \cdot \Pi(\mathbf{k} \times \Pi(\theta \mathbf{k}))\rangle+\lambda_{2} R\langle\theta \mathbf{k} \cdot \Pi(\mathbf{k} \times \Pi(\mathbf{u} \times \mathbf{k}))\rangle, \\
D(u)= & \|\nabla \mathbf{u}\|^{2}+\|\nabla \theta\|^{2}+\lambda_{2}\left\|\mathbf{u}{ }_{, z}\right\|^{2}+P_{0}\|\mathbf{u}\|^{2}+\lambda_{2} P_{0}\|\Pi(\mathbf{u} \times \mathbf{k})\|^{2} \\
N(u)= & -\frac{\lambda_{2}}{\phi}\left\langle( \mathbf { u } \cdot \nabla \mathbf { u } ) \cdot \Pi \left(\mathbf{k} \times \Pi(\mathbf{u} \times \mathbf{k})>-\frac{\lambda P_{r}^{1 / 2}}{\phi}\langle(\mathbf{u} \cdot \nabla \mathbf{u}) \cdot \Pi(\mathbf{k} \times \Pi(\theta \mathbf{k}))\rangle\right.\right. \\
& -\lambda P_{r}^{1 / 2}\langle(\mathbf{u} \cdot \nabla \theta)(\Pi(\mathbf{u} \times \mathbf{k}) \cdot \mathbf{k})\rangle, \\
F_{1}(u)= & 2 R\langle\nabla \theta \cdot \nabla w\rangle+2 R\langle\theta w\rangle, \\
D_{1}(u)= & \|\Delta \mathbf{u}\|^{2}+\|\Delta \theta\|^{2}+\|\nabla \mathbf{u}\|^{2}+\|\nabla \theta\|^{2}+P_{0}\|\nabla \mathbf{u}\|^{2}+P_{0}\|\mathbf{u}\|^{2} \\
N_{1}(u)= & -\frac{1}{\phi}\langle(\mathbf{u} \cdot \nabla \mathbf{u}) \cdot \Delta \mathbf{u}\rangle-P_{r}\langle(\mathbf{u} \cdot \nabla \theta) \Delta \theta\rangle .
\end{aligned}
$$

On using the properties of operator $\Pi$ in (6) and (7), we can write

$$
\Pi(\mathbf{k} \times \Pi(\mathbf{u} \times \mathbf{k}))=\mathbf{k} \times(\mathbf{u} \times \mathbf{k})-\mathbf{k} \times \nabla \zeta-\nabla \zeta_{1}
$$

with

$$
\begin{aligned}
& \Delta \zeta=\nabla \cdot(\mathbf{u} \times \mathbf{k})=v_{, x}-u_{, y}, \\
& \zeta_{, z}=0 \quad \text { at } z=0,1,
\end{aligned}
$$

and

$$
\begin{aligned}
& \Delta \zeta_{1}=\nabla \cdot(\mathbf{k} \times(\mathbf{u} \times \mathbf{k})-\mathbf{k} \times \nabla \zeta)=u_{, x}+v_{, y}, \\
& \zeta_{1, z}=0 \quad \text { at } z=0,1
\end{aligned}
$$

We also write

$$
\Pi(\mathbf{k} \times \Pi(\theta \mathbf{k}))=\mathbf{k} \times \Pi(\theta \mathbf{k})=\mathbf{k} \times(\theta \mathbf{k}-\nabla \tau)=-(\mathbf{k} \times \nabla) \tau
$$

with

$$
\Delta \tau=\theta_{, z}, \quad \tau_{, z}=0 \quad \text { at } z=0,1 .
$$

We then observe that

$$
\langle\theta \mathbf{k} \cdot \Pi(\mathbf{k} \times \Pi(\mathbf{u} \times \mathbf{k}))\rangle=-\left\langle\theta \zeta_{1, z}\right\rangle=\left\langle\theta_{, z} \zeta_{1}\right\rangle,
$$

and

$$
\begin{aligned}
\langle(\mathbf{u} \times \mathbf{k}) \cdot \Pi(\mathbf{k} \times \Pi(\theta \mathbf{k}))\rangle & =\left\langle\left(v \tau_{, y}+u \tau_{, x}\right)\right\rangle=-\left\langle\tau\left(u_{, x}+v_{, y}\right)\right\rangle \\
& =-\left\langle\tau \Delta \zeta_{1}\right\rangle=-\left\langle(\Delta \tau) \zeta_{1}\right\rangle=-\left\langle\theta_{, z} \zeta_{1}\right\rangle .
\end{aligned}
$$

With the choice

$$
\lambda_{2}=\lambda T P_{r}^{1 / 2} / R
$$


and using (31) and (32), we eliminate the last two terms in (20), which in general are not of one sign and may destabilize the solution. In view of the condition (18), we are led to choose

$$
\lambda=\xi T P_{r}^{1 / 2} / R, \quad \xi \in(0,1) .
$$

4. A priori estimation of $\dot{V}$. We now need an a priori estimate of $\dot{V}$ to find the sufficient conditions which ensure that the energy $V$ decays monotonically to zero.

We make the following observations:

$$
\begin{aligned}
N(u) \leq & \frac{\lambda_{2}}{\phi} \sup _{\Omega}|u|\|\nabla \mathbf{u}\|\|\Pi(\mathbf{u} \times \mathbf{k})\|+\frac{\lambda P_{r}^{1 / 2}}{\phi} \sup _{\Omega}|u|\|\nabla \mathbf{u}\|\|\Pi(\theta \mathbf{k})\| \\
& +\lambda P_{r}^{1 / 2} \sup _{\Omega}|u|\|\nabla \theta\|\|\Pi(\mathbf{u} \times \mathbf{k})\| \\
\leq & \frac{\lambda_{2} c}{\phi}\|\Delta \mathbf{u}\|\|\nabla \mathbf{u}\|\|\Pi(\mathbf{u} \times \mathbf{k})\|+\frac{\lambda c}{\phi} P_{r}^{1 / 2}\|\Delta \mathbf{u}\|\|\nabla \mathbf{u}\|\|\theta\| \\
& +\lambda c P_{r}^{1 / 2}\|\Delta \mathbf{u}\|\|\nabla \theta\|\|\Pi(\mathbf{u} \times \mathbf{k})\| \\
\leq & \left(\frac{c \lambda_{2}}{\phi}+\frac{c \lambda \lambda_{2}^{1 / 2}}{\phi}+c \lambda p_{r}^{1 / 2}\right) \frac{1}{\left(2 \lambda_{2}-2 \lambda^{2}\right)^{1 / 2}} D_{1} E^{1 / 2} \\
= & b D_{1} E^{1 / 2}
\end{aligned}
$$

where

$$
b=\frac{c \lambda_{2}}{\phi\left(2 \lambda_{2}-2 \lambda^{2}\right)^{1 / 2}}+\frac{c \lambda\left(\lambda_{2}\right)^{1 / 2}}{\phi\left(2 \lambda_{2}-2 \lambda^{2}\right)^{1 / 2}}+\frac{c \lambda P_{r}^{1 / 2}}{\left(2 \lambda_{2}-2 \lambda^{2}\right)^{1 / 2}}
$$

and

$$
\|\Pi(\mathbf{u} \times \mathbf{k})\| \leq\left(\frac{2}{\lambda_{2}-\lambda^{2}}\right)^{1 / 2} E^{1 / 2}, \quad\|\theta\| \leq\left(\frac{2 \lambda_{2}}{\left(\lambda_{2}-\lambda^{2}\right) P_{r}}\right)^{1 / 2} E^{1 / 2},
$$

have been used with

$$
c=\frac{3^{1 / 2}}{2^{1 / 4}\left[\pi h^{3}(\sqrt{2}-1)\right]^{1 / 2}}+\frac{40 h^{3 / 5}}{3}, \quad h=\min \left\{a_{1}, a_{2}, 1\right\}
$$

(cf. Galdi and Straughan (1985, p. 267)). We also observe that

$$
\begin{aligned}
N_{1}(u) & \leq \sup _{\Omega}|u|\left(\frac{1}{\phi}\|\nabla \mathbf{u}\|\|\Delta \mathbf{u}\|+P_{r}\|\nabla \theta\|\|\Delta \theta\|\right) \\
& \leq \sqrt{2} c\left(\frac{1}{\phi}+\frac{1}{2} P_{r}^{1 / 2}\right) E_{1}^{1 / 2} D_{1},
\end{aligned}
$$


and

$$
\begin{aligned}
F_{1}(u) & \leq 2 R\|w\|\|\Delta \theta\|+2 R\|w\|\|\theta\| \\
& \leq \frac{2}{\pi} R\left\|w{ }_{, z}\right\|\left(\|\Delta \theta\|+\frac{1}{\pi}\|\nabla \theta\|\right) \\
& \leq \frac{1}{2}\left(\|\nabla \theta\|^{2}+\|\Delta \theta\|^{2}\right)+2 R^{2} \frac{\pi^{2}+1}{\pi^{4}}\left\|\mathbf{u}_{, z}\right\|^{2} \\
& \leq \frac{1}{2} D_{1}+\frac{2 R^{2}\left(\pi^{2}+1\right)}{\lambda_{2} \pi^{4}} D,
\end{aligned}
$$

or

$$
\begin{aligned}
F_{1}(u) & \leq \frac{2 R}{\pi}\|\nabla w\|\left(\|\Delta \theta\|+\frac{1}{\pi}\|\nabla \theta\|\right) \\
& \leq \frac{1}{2} D_{1}+\frac{2 R^{2}\left(1+\pi^{2}\right)}{\pi^{4}} D
\end{aligned}
$$

where the Poincaré inequalities have been used in the derivation of the above results.

We now define

$$
m=\max _{\varphi} \frac{F}{D},
$$

where $\varphi$ is a set of the admissible functions,

$$
\varphi=\{\mathbf{u}, \theta \text { which are periodic in } x \text { and } y \text { and satisfy (1)-(4). }
$$

We suppose first that $T>1$; using (35)-(40) and (42) in (19) yields

$$
\begin{aligned}
\dot{V} \leq & \left\{(m-1)+\frac{2 \eta R^{2}\left(\pi^{2}+1\right)}{\lambda_{2} \pi^{4}}\right\} D-\frac{1}{2} \eta D_{1} \\
& +b D_{1} E^{1 / 2}+\sqrt{2} \eta c\left(\frac{1}{\phi}+\frac{1}{2} P_{r}^{1 / 2}\right) E_{1}^{1 / 2} D_{1} .
\end{aligned}
$$

On choosing

$$
\eta=\frac{\lambda_{2} \pi^{4}(1-m)}{4 R^{2}\left(\pi^{2}+1\right)}
$$

and setting

$$
\mathscr{D}=\frac{1}{2}\left[(1-m) D+\eta D_{1}\right]
$$

we conclude that

$$
\dot{V} \leq-\mathscr{D}\left(1-A V^{1 / 2}\right)
$$

where

$$
A=2 \frac{b}{\eta}+\frac{2 \sqrt{2} c\left(\frac{1}{\phi}+\frac{1}{2} P_{r}^{1 / 2}\right)}{\eta^{1 / 2}}
$$

By virtue of the Poincaré (Wirtinger) inequality (Galdi and Straughan, 1985), we also have

where

$$
\mathscr{D} \geq \frac{(1-m) \pi^{2}}{2 P^{*}} V
$$

$$
P^{*}= \begin{cases}1 & \text { if } P_{r} \leq 1, \\ P_{r} & \text { if } P_{r}>1\end{cases}
$$


Then from (47) we may show that

$$
V(t) \leq V(0) \exp \left\{-\frac{\pi^{2}(1-m)}{2 P^{*}}\left[1-A V^{1 / 2}(0)\right] t\right\},
$$

which means that $V(t) \rightarrow 0$ in a monotonic fashion as $t \rightarrow \infty$, whenever

$$
V(0)<A^{-2} \text {. }
$$

For $T \leq 1$ we use (41) to replace (40), then get the estimation in the form

$$
\begin{aligned}
\dot{V} \leq & \left.(m-1)+\frac{2 \eta R^{2}\left(\pi^{2}+1\right)}{\pi^{4}}\right\} D-\frac{1}{2} \eta D_{1} \\
& +b_{1} D_{1} E^{1 / 2}+\sqrt{2} \eta c\left(\frac{1}{\phi}+\frac{1}{2} P_{r}^{1 / 2}\right) E_{1}^{1 / 2} D_{1},
\end{aligned}
$$

where $b_{1}$ coincides with the value of $b$ for $T=1$. From (53), it follows that (51) holds with

$$
A=\frac{2 b_{1}}{\eta}+\frac{2 \sqrt{2} c\left(\frac{1}{\phi}+\frac{1}{2} P_{r}^{1 / 2}\right)}{\eta^{1 / 2}}
$$

and

$$
\eta=\frac{\pi^{4}(1-m)}{4 R^{2}\left(1+\pi^{2}\right)} .
$$

When $V(0)<A^{-2}$, we again obtain monotonic stability.

5. Variational problem. We now solve the variational problem associated with the maximum problem (42). The corresponding Euler-Lagrange equations are

$$
\begin{gathered}
m \Delta \mathbf{u}-m P_{0} \mathbf{u}+m \lambda_{2} \mathbf{u}_{, z z}-m \lambda_{2} P_{0}(\mathbf{k} \times \Pi(\mathbf{u} \times \mathbf{k}))+R \theta \mathbf{k} \\
-\frac{\lambda\left(1+P_{r}\right)}{2 \sqrt{P_{r}}} \mathbf{k} \times \operatorname{curl} \operatorname{curl}(\theta \mathbf{k})-\frac{\lambda P_{0} \sqrt{P_{r}}}{2} \mathbf{k} \times \Pi(\theta \mathbf{k}) \\
\left.+\frac{\lambda R}{2 \sqrt{P_{r}}}(\Pi(\mathbf{u} \times \mathbf{k}) \cdot \mathbf{k}) \mathbf{k}+\mathbf{k} \times \Pi(\mathbf{k} w)\right)=\nabla p, \\
\nabla \cdot \mathbf{u}=0, \\
m \Delta \theta+R w-\frac{\lambda\left(1+P_{r}\right)}{2 \sqrt{P_{r}}} \operatorname{curl} \operatorname{curl}(\mathbf{u} \times \mathbf{k}) \cdot \mathbf{k}-\frac{\lambda}{2} P_{0} \sqrt{P_{r}} \Pi(\mathbf{u} \times \mathbf{k}) \cdot \mathbf{k}=0 .
\end{gathered}
$$

Using the variables defined by (27) and (30) for $\zeta, \tau$ respectively, and a variable $\psi$ which is associated with $\Pi(\mathbf{k} w)$ in the form of

$$
\Pi(\mathbf{k} w)=\mathbf{k} w-\nabla \psi
$$

with

$$
\Delta \psi=w_{, z}, \quad \psi_{, z}=0 \quad \text { at } \quad z=0,1
$$


we can write (56) and (58) as

$$
\begin{aligned}
& m \Delta \mathbf{u}-m P_{0} \mathbf{u}+m \lambda \frac{T \sqrt{P_{r}}}{R} \mathbf{u}{ }_{, z z}-m \lambda \frac{T \sqrt{P_{r}}}{R} P_{0}(\mathbf{u}-w \mathbf{k}-(\mathbf{k} \times \nabla) \zeta) \\
& +R \theta \mathbf{k}+\lambda \frac{\left(1+P_{r}\right)}{2 \sqrt{P_{r}}}(\nabla \theta, z \times \mathbf{k})+\frac{\lambda}{2} P_{0} \sqrt{P_{r}}(\mathbf{k} \times \nabla \tau) \\
& +\frac{\lambda R}{2 \sqrt{P_{r}}}\left(-\zeta_{, z} \mathbf{k}-\mathbf{k} \times \nabla \psi\right)=\nabla p \\
& m \Delta \theta+R w-\frac{\lambda\left(1+P_{r}\right)}{2 \sqrt{P_{r}}} \omega_{, z}+\frac{\lambda}{2} P_{0}{\sqrt{P_{r}} \zeta_{, z}}=0
\end{aligned}
$$

where $\omega=(\nabla \times \mathbf{u}) \cdot \mathbf{k}$. Operating on $(60)$ with $\mathbf{k} \cdot \Delta$ curl and then with $\mathbf{k} \cdot \Delta$ curl curl, operating on (61) with $\Delta$, and using (33), we arrive at

$$
\begin{aligned}
& m \Delta^{2} \omega-m P_{0} \Delta \omega+m \frac{\lambda T{\sqrt{P_{r}}}}{R} \Delta \omega_{, z z}-m \frac{\lambda T \sqrt{P_{r}} P_{0}}{R} \omega_{, z z} \\
& -\lambda \frac{\left(1+P_{r}\right)}{2 \sqrt{P_{r}}} \Delta \Delta_{1} \theta_{, z}+\frac{\lambda P_{0}{\sqrt{P_{r}}}_{r} \Delta_{1} \theta_{, z}-\frac{\lambda R}{2 \sqrt{P_{r}}} \Delta_{1} w_{, z}=0}{2} \\
& m \Delta^{3} w-m P_{0} \Delta^{2} w+m \frac{\lambda T \sqrt{P_{r}}}{R} \Delta^{2} w_{, z z}-m \frac{\lambda T \sqrt{P_{r}} P_{0}}{R} \Delta w_{, z z} \\
& +R \Delta \Delta_{1} \theta+\frac{\lambda R}{2 \sqrt{P_{r}} \Delta_{1} \omega_{, z}=0,} \\
& m \Delta^{2} \theta+R \Delta w-\frac{\lambda\left(1+P_{r}\right)}{2 \sqrt{P_{r}}} \Delta \omega_{, z}+\frac{\lambda}{2} P_{0}{\sqrt{P_{r}} \omega_{, z}=0 .}=0 .
\end{aligned}
$$

We remark that if we set $P_{0}=0$ in the above system of equations (62)-(64), these reduce to the same set of equations as studied by Galdi and Padula (1990). Eliminating variables $\theta$ and $\omega$ yields

$$
\begin{aligned}
& \Delta\left(\Delta-P_{0}\right)\left[\Delta+\lambda T \sqrt{P_{r}} / R\left(D^{2}-P_{0}\right)\right] \\
& \cdot\left\{\left(\frac{\lambda\left(1+P_{r}\right)}{2 \sqrt{P_{r}}} \Delta-\frac{\lambda P_{0} \sqrt{P_{r}}}{2}\right)^{2} \Delta_{1} D^{2}-m^{2} \Delta^{2}\left(\Delta-P_{0}\right)\left(\Delta+\frac{\lambda T \sqrt{P_{r}}}{R} D^{2}\right)\right\} w \\
& +\left\{-\frac{\lambda^{2} R^{2}}{4 P_{r}} \Delta^{2} \Delta_{1}^{2} D^{2}+R^{2} \Delta^{2} \Delta_{1}\left(\Delta-P_{0}\right)\left(\Delta+\frac{\lambda T \sqrt{P_{r}}}{R} D^{2}\right)\right\} w=0,
\end{aligned}
$$

where $D=\partial / \partial z$ and $\Delta_{1}=\partial^{2} / \partial x^{2}+\partial^{2} / \partial y^{2}$. 
On employing standard normal mode analysis we may write $w=W(z) e^{2 i\left(a_{1} x+a_{2} y\right)}$. The boundary conditions (4) together with the equations (1)-(3) can produce $D^{(2 l)} W$ $=0$ at $z=0,1$ for $l=1,2, \ldots$. This indicates that $W(z)$ can be assumed to have the form $W(z)=H \sin r \pi z$, for $r=1,2, \ldots$ and $H$ an arbitrary constant. Substituting these functions into equation (65) and noting (34), we obtain

$$
\begin{aligned}
- & \left(r^{2} \pi^{2}+a^{2}\right)\left(r^{2} \pi^{2}+a^{2}+P_{0}\right)\left(r^{2} \pi^{2}+a^{2}+\frac{\xi T^{2} P_{r}}{R^{2}} r^{2} \pi^{2}\right) \\
& \times\left\{\frac{\xi^{2} T^{2}}{4 R^{2}}\left[\left(1+P_{r}\right)\left(r^{2} \pi^{2}+a^{2}\right)+P_{0} P_{r}\right]^{2} r^{2} \pi^{2} a^{2}\right. \\
- & \left.m^{2}\left(r^{2} \pi^{2}+a^{2}\right)^{2}\left(r^{2} \pi^{2}+a^{2}+P_{0}\right)\left(r^{2} \pi^{2}+a^{2}+\frac{\xi T^{2} P_{r}}{R^{2}} r^{2} \pi^{2}\right)\right\} \\
+ & \left\{\frac{\xi^{2} T^{2}}{4}\left(r^{2} \pi^{2}+a^{2}\right)^{2} r^{2} \pi^{2} a^{4}-R^{2} a^{2}\left(r^{2} \pi^{2}+a^{2}\right)^{2}\left(r^{2} \pi^{2}+a^{2}+P_{0}\right)\right. \\
& \left.\times\left(r^{2} \pi^{2}+a^{2}+\frac{\xi T^{2} P_{r}}{R^{2}} r^{2} \pi^{2}\right)\right\}=0,
\end{aligned}
$$

where $a^{2}=4\left(a_{1}^{2}+a_{2}^{2}\right)$.

For convenience, we rewrite the above equation as a polynomial equation in $R^{2}$ in the form

$$
p\left(R^{2}, m\right)=R^{6}+A_{1}(m) R^{4}+B_{1}(m) R^{2}+C_{1}(m)=0,
$$

where

$$
\begin{aligned}
A_{1}(m)=- & m^{2} r^{4} \pi^{4} \frac{(1+\alpha)^{2}(1+\alpha+\beta)}{\alpha}+\frac{\xi T^{2} P_{r}}{(1+\alpha)} \\
& -\frac{\xi^{2} T^{2}}{4} \frac{\alpha}{(1+\alpha)(1+\alpha+\beta)}, \\
B_{1}(m)=r^{4} \pi^{4}\{ & -2 m^{2} \xi T^{2} P_{r} \frac{(1+\alpha)(1+\alpha+\beta)}{\alpha} \\
& \left.+\frac{\xi^{2} T^{2}}{4} \frac{\left[\left(1+P_{r}\right)(1+\alpha)+\beta P_{r}\right]^{2}}{(1+\alpha)}\right\}, \\
C_{1}(m)=r^{4} \pi^{4}\{ & -m^{2} \xi^{2} T^{4} P_{r}^{2} \frac{(1+\alpha+\beta)}{\alpha} \\
& \left.+\frac{\xi^{3} T^{4} P_{r}}{4} \frac{\left[\left(1+P_{r}\right)(1+\alpha)+\beta P_{r}\right]^{2}}{(1+\alpha)^{2}}\right\},
\end{aligned}
$$

with

$$
\alpha=a^{2} /\left(r^{2} \pi^{2}\right), \quad \beta=P_{0} /\left(r^{2} \pi^{2}\right) .
$$


Choosing

$$
\xi<\frac{4 P_{r}(1+\alpha)^{2}(1+\alpha+\beta)}{\alpha\left[\left(1+P_{r}\right)(1+\alpha)+\beta P_{r}\right]^{2}}
$$

and denoting $A=A_{1}(1), B=B_{1}(1), C=C_{1}(1)$, we observe that there is one and only one positive number $R_{c}^{2}$ satisfying the equation

$$
P\left(R^{2}, 1\right)=R^{6}+A R^{4}+B R^{2}+C=0
$$

and

$$
P\left(R^{2}, 1\right)<0 \text { for any } R^{2}<R_{c}^{2}
$$

because both $B$ and $C$ are negative under the assumption of (70).

Moreover, a straightforward calculation shows that

$$
\frac{d A_{1}(m)}{d m}<A, \quad \frac{d B_{1}(m)}{d m}<B, \quad \frac{d C_{1}(m)}{d m}<C
$$

for any $m \geq 1$, where the restriction in (70) was used to derive the first inequality in (73).

Therefore, a comparison shows for any $R^{2}<R_{c}^{2}$

$$
\frac{d P\left(R^{2}, m\right)}{d m}<P\left(R^{2}, 1\right)<0
$$

for any $m \geq 1$. This implies that $P\left(R^{2}, m\right)<0$ if $m \geq 1$ for any $R^{2}<R_{c}^{2}$, while $R_{c}^{2}$ is a positive root of equation (71) at the critical argument $m=1$. We thus conclude that $R<R_{c}$ implies $m<1$. We also remark here that (70) will certainly be satisfied if the further choice

$$
\xi<\frac{4 P_{r}(1+\alpha)}{\alpha\left(1+P_{r}\right)^{2}(1+\beta)}
$$

is required.

With the restriction in (75), it is also straightforward to verify that $r=1 \mathrm{mini}$ mizes $R_{c}$. Actually taking differentiation with respect to $r^{2}$ on both sides of (71), we reduce the equation to

$$
\left(3 R_{c}^{4}+2 A R_{c}^{2}+B\right) R_{c}^{2^{\prime}}=-A^{\prime} R_{c}^{4}-B^{\prime} R_{c}^{2}-C^{\prime}
$$

where the prime denotes differentiation with respect to $r^{2}$. An inspection of expres- 
sions for $A, B, C$ and the observations

$$
\begin{aligned}
A^{\prime}= & \frac{\partial A}{\partial r^{2}}+\frac{\partial A}{\partial \beta} \frac{\partial \beta}{\partial r^{2}}=\frac{\partial A}{\partial r^{2}}+\frac{\partial A}{\partial \beta}\left(-\frac{\beta}{r^{2}}\right) \\
= & -2 r^{2} \pi^{4} \frac{(1+\alpha)^{2}(1+\alpha+\beta)}{\alpha}+r^{2} \pi^{4} \frac{(1+\alpha)^{2} \beta}{\alpha}-\frac{\xi^{2} T^{2} \alpha \beta}{(1+\alpha)(1+\alpha+\beta)^{2} r^{2}} \\
= & -r^{2} \pi^{4} \frac{(1+\alpha)^{2}(1+\alpha+\beta)}{\alpha}-r^{2} \pi^{4} \frac{(1+\alpha)^{3}}{\alpha}-\frac{\xi^{2} T^{2} \alpha \beta}{(1+\alpha)(1+\alpha+\beta)^{2} r^{2}}<0, \\
B^{\prime}= & \frac{\partial B}{\partial r^{2}}+\frac{\partial B}{\partial \beta}\left(-\frac{\beta}{r^{2}}\right) \\
= & r^{2} \pi^{4}\left\{-2 \xi T^{2} P_{r} \frac{(1+\alpha)(1+\alpha+\beta)}{\alpha}+\xi^{2} T^{2} \frac{\left[\left(1+P_{r}\right)(1+\alpha)+\beta P_{r}\right]^{2}}{4(1+\alpha)}\right\} \\
& +r^{2} \pi^{4}\left\{-2 \xi T^{2} P_{r} \frac{(1+\alpha)^{2}}{\alpha}+\frac{\xi^{2} T^{2}}{4}\left(1+P_{r}\right)^{2}(1+\alpha)-\frac{\gamma^{2} \pi^{2} \xi^{2} T^{2}}{4(1+\alpha)} \beta^{2} P_{r}^{2}\right\}, \\
C^{\prime}= & \frac{\partial C}{\partial r^{2}}+\frac{\partial C}{\partial \beta}\left(-\frac{\beta}{r^{2}}\right) \\
= & r^{2} \pi^{4}\left\{-\xi^{2} T^{4} P_{r}^{2} \frac{(1+\alpha+\beta)}{\alpha}+\frac{\xi^{3} T^{4} P_{r}}{4} \frac{\left[\left(1+P_{r}\right)(1+\alpha)+\beta P_{r}\right]^{2}}{(1+\alpha)^{2}}\right\} \\
& +r^{2} \pi^{4}\left\{-\xi^{2} T^{4} P_{r}^{2} \frac{(1+\alpha)}{\alpha}+\frac{\xi^{3} T^{4} P_{r}}{4}\left(1+P_{r}\right)^{2}\right\}-r^{2} \pi^{4} \frac{\xi^{3} T^{4} P_{r}}{4} \frac{\beta^{2} P_{r}^{2}}{(1+\alpha)^{2}}
\end{aligned}
$$

together with the restriction of the choice in (75) show that the right-hand side of (76) is positive vrhile the term in the brackets on the left side is positive. We thus conclude that $\partial R_{c}^{2} / \partial\left(r^{2}\right)>0$ and the minimum is attained at $r=1$. The critical energy bound will be defined by

$$
R_{e}^{2}=\min _{\alpha} \max _{\xi} R_{c}^{2}\left(\alpha, \xi, P_{0}, P_{r}, T^{2}\right) .
$$

Equation (78) has been solved numerically, and Tables 1 and 2 give several values of $R_{e}^{2}$ as a function of $T$ and $P_{0}$. The values of $P_{r}$ are, however, fixed and, for comparison purposes, are taken to be the same as those of Galdi and Padula (1990). Since we are concerned with high porosity systems only, comparatively small values for $P_{0}$ are considered. Except with a minor difference in one of the values, our calculations completely agree with the results given by Galdi and Padula (1990) when the parameter $P_{0}=0$. We point out that for the low porosity system, $P_{0}$ becomes very large and the coupling parameter $\xi$ has to be very small due to the choice of (75). In this case $R_{e}^{2}$ tends to $P_{0} \pi^{2} / 4$, which is the critical value without rotation.

From the tables we note that as $T$ increases, the critical energy bound also increases. For the range of values of the Taylor number in the tables, the effect of porosity is to stabilize the system; that is, as $P_{0}$ increases so does the value of $R_{e}^{2}$. 
Table 1. Energy Critical Rayleigh Numbers

\begin{tabular}{|c|c|c|c|c|}
\hline \multicolumn{5}{|c|}{$P_{r}=6.8$} \\
\hline$T^{2}$ & $\begin{array}{r}P_{0}=0.0 \\
R_{e}^{2}\end{array}$ & $\begin{array}{r}P_{0}=0.1 \\
R_{e}^{2}\end{array}$ & $\begin{array}{r}P_{0}=1.0 \\
R_{e}^{2}\end{array}$ & $R_{G}^{2}$ \\
\hline 0 & 657.511 & 661.950 & 701.690 & 657.511 \\
\hline 10 & 672.381 & 676.697 & 715.453 & 672.609 \\
\hline 50 & 723.235 & 727.254 & 763.453 & 723.236 \\
\hline 100 & 775.105 & 778.921 & 813.305 & 775.104 \\
\hline 200 & 858.523 & 862.115 & 894.579 & 858.514 \\
\hline 300 & 926.967 & 930.251 & 961.702 & 926.750 \\
\hline 400 & 985.907 & 989.332 & 1020.11 & 1001.92 \\
\hline 1000 & 1249.95 & 1253.02 & 1282.44 & 1249.83 \\
\hline
\end{tabular}

$R_{G-P}^{2}$-results obtained by Galdi and Padula

Table 2. Energy Critical Rayleigh Numbers

\begin{tabular}{|r|r|r|r|r|}
\multicolumn{5}{c|}{$P_{r}=200$} \\
$T^{2}$ & $P_{0}=0.0$ & $P_{0}=0.1$ & $P_{0}=1.0$ & $P_{0}=1.0$ \\
& $R_{e}^{2}$ & $R_{e}^{2}$ & $R_{e}^{2}$ & $R_{G-P}^{2}$ \\
\hline 0 & 657.511 & 661.950 & 701.690 & 657.511 \\
10 & 676.37 & 680.637 & 718.966 & 676.75 \\
50 & 738.95 & 742.805 & 777.592 & 738.94 \\
100 & 800.99 & 804.574 & 836.988 & 800.99 \\
250 & 940.09 & 943.304 & 972.407 & \\
500 & 1106.76 & 1109.74 & 1136.60 & \\
1000 & 1347.78 & 1350.54 & 1375.55 & 1347.78 \\
2000 & 1690.67 & 1693.28 & 1716.90 & \\
5000 & 2369.71 & 2371.79 & 2394.14 & \\
\hline
\end{tabular}

$R_{G-P}^{2}$-results obtained by Galdi and Padula

Acknowledgment. The work reported in this paper has been supported in part by an OGS Scholarship to Y. Qin and in part by Grant No. A7728 of NSERC of Canada. The authors gratefully acknowledge the support thus received.

\section{REFERENCES}

[1] S. Chandrasekhar, Hydrodynamic and Hydromagnetic Stability, Oxford University Press, Oxford, 1961

[2] S. H. Davis, On the principle of exchange of stabilities, Proc. Roy. Soc. London Ser. A 310, 341-358 (1969)

[3] G. P. Galdi, Nonlinear stability of the magnetic Bénard problem via a generalized energy method, Arch. Rational Mech. Anal. 87, 167-186 (1985)

[4] G. P. Galdi and B. Straughan, Exchange of stabilities, symmetry and nonlinear stability, Arch. Rational Mech. Anal. 89, 211-228 (1985a)

[5] G. P. Galdi and B. Straughan, A nonlinear analysis of the stabilizing effect of rotation in the Benard problem, Proc. Roy. Soc. London Ser. A 402, 257-283 (1985b) 
[6] G. P. Galdi and M. Padula, A new approach to energy theory in the stability of fluid motion, Arch. Rational Mech. Anal. 110, 187-286 (1990)

[7] D. D. Joseph, On the stability of Boussinesq equations, Arch. Rational Mech. Anal. 20, 59-71 (1965)

[8] D. D. Joseph, Nonlinear stability of Boussinesq equations by the method of energy, Arch. Rational Mech. Anal. 22, 163-184 (1966)

[9] D. D. Joseph, Stability of fluid motion. Vols. I, II, Springer-Verlag, Berlin, Heidelberg and New York, 1976

[10] G. Mulone and S. Rionero, On the nonlinear stability of the rotating Bénard problem via the Lyapunov direct method, J. Math. Anal. Appl. 144, 109-127 (1989)

[11] W. McF. Orr, The stability or instability of the steady motions of perfect liquid and of a viscous liquid, Proc. Roy. Irish Acad. A27, 9-68 and 69-138 (1907)

[12] Y. Qin and P. N. Kaloni, Steady convection in a porous medium based upon the Brinkman model, IMA J. Appl. Math. 35, 85-95 (1992)

[13] S. Rionero, Metodi variazionali per la stabilità asintotica in media in magnetoidrodinamica, Ann. Mat. Pura Appl. (4) 78, 339-364 (1968)

[14] S. Rionero and B. Straughan, Convection in a porous medium with internal heat source and variable gravity effects, Internat. J. Engrg. Sci. 28, 497-503 (1990)

[15] J. Serrin, On the stability of viscous fluid motion, Arch. Rational Mech. Anal. 3, 1-13 (1959)

[16] K. Walker and G. M. Homsy, A note on convective instability in Boussinesq fluids and porous media, J. Heat Transfer 99, 338-339 (1977)

[17] D. R. Westbrook, The stability of convective flow in a porous medium, Phys. Fluids 12, 1547-1551 (1969) 